\title{
AVALIAÇÃO DA SENSIBILIDADE E ESPECIFICIDADE DOS EXAMES CITOPATOLÓGICO E COLPOSCÓPICO EM RELAÇ̃OO AO EXAME HISTOLÓGICO NA IDENTIFICAÇÃO DE LESÖES INTRA-EPITELIAIS CERVICAIS
}

\author{
Felipe Francisco Bondan Tuon*, Márcio Sommer Bittencourt, Maria Alice Panichi, Álvaro Piazetta Pinto \\ Serviço de Patologia do Trato Genital Inferior e Colposcopia, Hospital e Maternidade Santa Brígida \& \\ Serviço de Anatomia Patológica do Hospital de Clínicas da Universidade Federal do Paraná, Curitiba, PR.
}

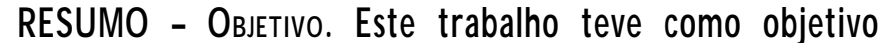
avaliar a concordância dos exames citológico e colposcópico com 0 resultado da análise histológica, obtida a partir de biópsia colpodirigida.

Métodos. Foram selecionadas 80 pacientes do ambulatório de colposcopia do Hospital e Maternidade Santa Brígida de Curitiba, PR. As pacientes foram encaminhadas à colposcopia segundo os seguintes critérios: 1) exames citológicos prévios com resultado anormal, 2) dados clínicos indicativos de alteração ou 3) lesões suspeitas ao exame ginecológico. A análise da significância estatística entre os resultados dos exames foi realizada por meio do teste de Qui-quadrado e foram determinados os valores correspondentes à sensibilidade, especificidade, valor preditivo negativo e valor preditivo positivo de cada método.

Resultados. As pacientes apresentaram idade média de 30,2 anos $( \pm 10,9)$. A capacidade de identificação da presença de lesão na citologia com relação à histologia foi de $50 \%$. A especificidade da citologia foi de $77 \%$, a sensibilidade de $41 \%$, o valor preditivo positivo de $74 \%$ e o valor preditivo negativo de $45 \%$. A capacidade da colposcopia em identificar a presença de lesão foi de $51 \%$. A sensibilidade foi de $96 \%$, a especificidade de $19 \%$, o valor preditivo positivo de $65 \%$ e 0 valor preditivo negativo de $75 \%$. A capacidade dos dois métodos associados de detectar a presença de lesões foi de $63 \%$.

ConclusöEs. A citologia mostrou-se um exame de alta especificidade, enquanto que a colposcopia das pacientes selecionadas apresentou alta sensibilidade. A associação de colposcopia ao screening feito pela citologia, em pacientes selecionadas, aumenta muito a acurácia do diagnóstico das lesões precursoras do carcinoma do colo uterino.

UnItermos: Neoplasia intra-epitelial. Citopatologia. Papanicolau. Colposcopia. Estudo comparativo. Histologia.

\section{INTRODUÇÃO}

As lesões intra-epiteliais escamosas do colo do útero são comprovadamente precursoras do carcinoma invasivo cervical'. Sua identificação precoce é de grande importância na terapêutica e prognóstico das pacientes. Vários exames são utilizados na avaliação destas lesões. Dentre eles, destaca-se a utilização do exame citológico acompanhado de colposcopia.

*Correspondência

R. Prof. Paulo Assunção, 903 - casa 3 81.540 - 260 - Curitiba - PR
O exame citológico de Papanicolaou é o método de excelência na avaliação do grau de alteração celular do epitélio escamoso cervical, e tem ajudado a diminuir drasticamente a incidência de câncer de colo uterino ${ }^{2}$. Para a classificação dos resultados dos exames, vários sistemas de nomenclatura podem ser utilizados. O sistema de Bethesda ${ }^{3}$ é o mais utilizado, classificando as anormalidades do epitélio escamoso cervical em lesão de baixo grau (LIBG), lesão de alto grau (LIAG), atipias celulares de significado indeterminado (ASCUS) e carcinoma invasor.

Nos casos em que há alteração do padrão normal do epitélio cervical, encaminham-se as pacientes para avaliação colposcópica ${ }^{4,5}$. A associação destes métodos mostra-se de grande importância diagnóstica ${ }^{6}$. Para uma maior acurácia diagnóstica, em casos de identificação de lesão durante a colposcopia, costuma-se fazer uma punção biópsia para diagnóstico histológico da anormalidade?.

Apesar da correlação entre os diagnósticos citopatológico, histopatológico e colposcópico estar estabelecida, vários trabalhos apontam controvérsias entre os resultados destas avaliações na dependência dos critérios e classificações utilizados ${ }^{6,8,9,10}$. 
Este trabalho tem como objetivo avaliar a acurácia dos exames citológico e colposcópico na detecção de lesões intraepiteliais escamosas. Exames histológicos dos produtos de biópsias colpodirigidas, de pacientes selecionadas para exame colposcópico, foram utilizados como o indicador definitivo da presença ou ausência de doença cervical.

\section{Métodos}

O presente estudo foi realizado no Hospital e Maternidade Santa Brígida de Curitiba, no estado do Paraná, no período de Junho de 1997 a Dezembro de 1998. As pacientes foram inicialmente atendidas pelo Serviço de Residência Médica de Ginecologia e Obstetrícia deste Hospital. De todas as pacientes que procuraram o serviço durante este período, foram encaminhadas ao ambulatório de colposcopia aquelas que apresentaram exame citológico anterior ou concomitante com resultado anormal, alterações ao exame de genitália externa ou especular, história de infecção pelo papilomavírus humano (HPV) e outras doenças sexualmente transmissíveis (DSTs). Pacientes com história de lesão de trato inferior e cauterizações prévias também foram avaliadas colposcopicamente. Foram incluídas no presente estudo todas as pacientes que se submeteram a biopsia, independente do resultado da análise histopatológica. Um segundo critério de seleção foi a satisfatoriedade dos exames citopatológico e colposcópico.

Resultados dos exames citopatológicos destas pacientes foram obtidos. Estes resultados foram reclassificados em negativos, lesões de baixo grau e lesões de alto grau, segundo a nomenclatura de Bethesda. Foram descartados os casos com diagnóstico de ASCUS. Após 60 dias, estas pacientes foram submetidas a col- poscopia. A classificação colposcópica utilizada no estudo seguiu a da Internacional Federation for Cervical Pathology and Colposcopy (IFCPC) determinada em Roma, no ano de 1990". De acordo com esta classificação, são considerados achados normais o epitélio pavimentoso original, o epitélio cilíndrico e zona de transformação normal. Os critérios de anormalidade são divididos, de acordo com a sua gravidade, em lesões maiores e lesões menores. São consideradas lesões menores as que apresentam epitélio acetobranco fino, mosaico regular, leucoplasia fina e vasos típicos. Já as lesões maiores caracterizam-se por epitélio branco espessado, mosaico irregular, pontilhado irregular, leucoplasia espessada e vasos atípicos. Durante a colposcopia, estas pacientes foram submetidas à biópsia dirigida, sendo o material enviado para avaliação histológica, corado por Hematoxilina-Eosina e classificado como negativo, lesão intra-epitelial de baixo e alto grau. Os resultados descritos nos exames colposcópico, histológico e citológico originais foram reclassificados de acordo com as categorias acima especificadas.

A correlação dos resultados da citologia e colposcopia foi feita em relação à histopatologia, por este ser o exame que providência o diagnóstico definitivo destas lesões ${ }^{12}$. Correlacionou-se os resultados dos exames citológicos e colposcópicos associados (presença ou não de lesão) com a histologia. Para essa associação, os exames com citologia negativa e colpocopia negativa foram agrupados sob a denominação "ausência de lesão". A "presença de lesão" foi considerada nos casos com citologia e colposcopia positivos, citologia negativa com colposcopia positiva, ou citologia positiva e colposcopia negativa. Foram calculados a sensibilidade, especificidade, o valor preditivo positivo (VPP) e valor preditivo negativo
(VPN) para os exames citológico, colposcópico e para a associação dos dois métodos, conforme descrito acima.

\section{Resultados}

A idade das pacientes variou de 15 a 67 anos, com média de 30,2 ( $\pm 10,9)$ anos. Quinze pacientes eram nulíparas, 28 eram primíparas, 35 eram multíparas (2 a 6 gestações), enquanto que em um caso, esta informação não constava no prontuário.

Quando correlacionados os resultados da citologia com os da histologia, verificou-se uma maior associação entre os casos de LIAG (79\%), ou seja, a citologia acertou o diagnóstico mais vezes quando a lesão era de alto grau. Nos casos onde o exame citológico foi negativo ou LIBG, a dispersão foi maior, mostrando que nestas categorias, o erro no diagnóstico citopatológico foi maior, havendo correlação com a histologia em apenas 45\% e $38 \%$ dos casos, respectivamente (Tabela I). Utilizando a análise histológica a partir de biópsias colpodirigidas como padrão ouro, obteve-se na citologia, uma especificidade de $77 \%$ e uma sensibilidade de $41 \%$. O VPP e o VPN foram de $74 \%$ e $45 \%$, respectivamente.

Quando correlacionados os resultados da colposcopia com os da histologia, verificou-se uma maior associação entre os casos negativos (75\%), isto é, a colposcopia acertou mais vezes quando não havia lesão. Observou-se também uma boa correlação entre as lesões maiores diagnosticadas colposcopicamente e as LIAG diagnosticadas a partir da análise histológica das biópsias (69\%). Nos casos onde a colposcopia detectou lesão menor, a dispersão foi maior, mostrando que nesta categoria o erro foi maior, encontrando-se correlação com o diagnóstico de LIBG a partir da histologia em apenas 
Tabela 1 - Correlação entre a citologia cérvico-vaginal e a histologia por biópsia colpodirigida de 80 pacientes

\begin{tabular}{lcccc}
\hline CITOLOGIA & \multicolumn{4}{c}{ HISTOLOGIA } \\
\cline { 2 - 5 } & Alto Grau & Baixo Grau & Negativo \\
Alto Grau & $11(79 \%)$ & $0(0 \%)$ & $3(21 \%)$ & $\mathbf{1 4}$ \\
Baixo Grau & $4(31 \%)$ & $5(38 \%)$ & $4(31 \%)$ & $\mathbf{1 3}$ \\
Negativo & $7(13 \%)$ & $22(42 \%)$ & $24(45 \%)$ & $\mathbf{5 3}$ \\
& $\mathbf{2 2}$ & $\mathbf{2 7}$ & $\mathbf{3 1}$ & $\mathbf{8 0}$ \\
\hline
\end{tabular}

Tabela 2 - Correlação entre a colposcopia e a histologia por biópsia colpodirigida de 80 pacientes

\begin{tabular}{lcccc}
\hline CITOLOGIA & \multicolumn{4}{c}{ HISTOLOGIA } \\
\cline { 2 - 5 } & Alto Grau & Baixo Grau & Negativo \\
Alto Grau & $1 \mid(69 \%)$ & $2(13 \%)$ & $3(19 \%)$ & $\mathbf{1 6}$ \\
Baixo Grau & $10(18 \%)$ & $24(43 \%)$ & $22(39 \%)$ & $\mathbf{5 6}$ \\
Negativo & $\mid(13 \%)$ & $1(13 \%)$ & $6(75 \%)$ & $\mathbf{8}$ \\
& $\mathbf{2 2}$ & $\mathbf{2 7}$ & $\mathbf{3 1}$ & $\mathbf{8 0}$ \\
\hline
\end{tabular}

\begin{tabular}{|c|c|c|c|}
\hline $\begin{array}{l}\text { CITOLOGIA E } \\
\text { COLPOSCOPIA }\end{array}$ & \multicolumn{3}{|c|}{ HISTOLOGIA } \\
\hline $\begin{array}{l}\text { Presença de Lesão } \\
\text { Ausência de Lesão }\end{array}$ & $\begin{array}{c}\text { Presença } \\
\text { de Lesáo } \\
50(63 \%) \\
\mid(\mid \%) \\
51\end{array}$ & $\begin{array}{c}\text { Ausência } \\
\text { de Lesão } \\
26(33 \%) \\
3(4 \%) \\
29\end{array}$ & $\begin{array}{c}76 \\
4 \\
80\end{array}$ \\
\hline
\end{tabular}

43\% dos casos (Tabela 2). A sensibilidade do método colposcópico foi $96 \%$ e a especificidade de 19\%. O VPP e o VPN da colposcopia foram de $65 \%$ e $75 \%$ respectivamente.

Correlacionando a colposcopia e a citologia em relação à histologia, somando-se as três categorias analisadas, obteve-se associação positiva em respectivamente $51 \%$ e $50 \%$ dos casos.

A correlação do método citológico associado ao colposcópico foi calculada em relação à histologia. Esta associação levou em consideração a presença ou não de lesão (Tabela 3). A sensibilidade do método citológico associado ao colposcópico foi 98\% e a especificidade 10\%. 0 VPP da associação entre a citologia e a colposcopia foi $66 \%$ e o VPN foi $75 \%$.

Quando associamos a citologia e a colposcopia em uma categoria por ausência ou presença de lesão, a correlação com a histologia aumentou para $66 \%$.

\section{Discussão}

A prevenção e o diagnóstico precoce correspondem às únicas maneiras de se reduzir a morbidade e mortalidade decorrentes das neoplasias ${ }^{25}$. A idéia de triagem para detecção precoce do câncer do colo do útero foi aceita a partir do desenvolvimento de técnicas de citologia exfoliativa pelo Dr. George Papanicolaou, em 1941. A citologia pelo método Papanicolaou apresenta uma boa sensibilidade e alta especificidade quando utilizada em populações como método de triagem ${ }^{15}$. Entretanto, a sensibilidade e especificidade do método se reduzem quando são analizados em pacientes com altera- ções cervicais ${ }^{10}$. A principal finalidade do método citológico é o rastreamento populacional das lesões intra-epiteliais, as quais podem ser melhor definidas a partir de exame colposcópico e estudo anatomopatológico de material proveniente de biópsia, CAF ou cirurgia.

Em nosso estudo, foi encontrado um valor de sensibilidade para citologia igual a $41 \%$. Este valor está dentro dos padrões para uma amostra de pacientes que já apresentam alguma alteração ${ }^{13}$. Estes mesmos conceitos se aplicam para o VPP e a especificidade que, se compararmos com os valores aplicados na população geral, estão bastante diminuídos. Todavia, quando calculamos estes parâmetros em populações com alterações ao nível de colo de útero, os valores de $77 \%$ e $74 \%$ para a especificidade e VPP de nosso estudo são coerentes.

A sensibilidade do método colposcópico encontrada em nosso estudo foi de $96 \%$. Este valor mostra que o método identificou alterações com eficiência, e está dentro dos padrões internacionais ${ }^{14,18}$. Por outro lado, a especificidade encontrada foi de 19\%. Isto significa que a colposcopia errou muito o diagnóstico. Este valor é difícil de ser questionado, uma vez que as alterações classificadas pela colposcopia não têm uma relação direta com a histologia como têm a citologia. Quando se associou, em nosso estudo, os resultados da citologia com a colposcopia, a sensibilidade aumentou para 98\%. Reafirma este fato o papel da colposcopia como um bom método para o diagnóstico de alterações do colo, quando associado a citopatologia.

No presente estudo, a correlação entre o diagnóstico citológico e histológico ocorreu em apenas 50\% $(40 / 80)$ das pacientes. Heatley et al. ${ }^{10}$ encontraram achados similares ao comparar resultados de citologia 
cérvico-vaginal com a histologia por biópsia colpodirigida. Valor semelhante foi encontrado por Parham utilizando mesma técnica. Estes autores encontraram, respectivamente, $49 \%$ e 55\% de concordância e concluíram ser a análise da histologia o método mais preciso para a estimativa do grau da lesão intra-epitelial.

A correlação entre a colposcopia e a histologia foi semelhante à concordância entre a citologia e histologia. $O$ valor de $51 \%$ está um pouco abaixo do observado na literatura internacional|' ${ }^{16}$. Este baixo valor de correlação em nosso estudo ocorreu principalmente pela discrepância das lesões classificadas como lesão menor. Há uma tendência dos colposcopistas em diagnosticá-las como negativas, e isso pode ter implicações muito sérias no tratamento destas pacientes ${ }^{19}$. Este erro é diminuído quando temos uma discordância entre o laudo citológico e a avaliação colposcópica, o que leva o colposcopista a realizar uma biópsia dirigida e confirmar o diagnóstico pela histologia ${ }^{20}$. Este erro no diagnóstico das lesões de baixo grau continua cada vez mais sério à medida que este se torna o tipo histológico mais freqüentemente encontrado nas populações atuai ${ }^{21}$. Os testes moleculares para a tipagem do HPV têm aumentado sua popularidade entre os ginecologistas e pato$\operatorname{logistas}^{28}$. Estes testes caracterizam-se por identificar e detectar o subtipo de HPV responsável pela alteração epitelial. Através destes exames é possível classificar o HPV em alto risco e baixo risco. Sendo as lesões de baixo grau as de maior dificuldade diagnóstica e terapêutica, a identificação do subtipo de HPV seria importante nestes casos ${ }^{2,27}$.

Outro motivo que justifica as baixas correlações observadas em nosso estudo baseia-se no material utilizado como gold standard. Skehan et al. já mostraram em seus trabalhos que a biópsia dirigida pela colposcopia apresenta um número muito grande de falso-negativos, podendo chegar até $54 \%$, quando comparada com material proveniente de conização22. Quando se associou o método colposcópico ao citológico em nosso estudo, obteve-se uma correlação de $66 \%$ em relação a histologia. Isto comprovou que a associação dos métodos citológico e colposcópico apresenta uma maior eficiência do que quando utilizados isoladamente.

$\mathrm{Na}$ presença das lesões de alto grau, o diagnóstico é de fundamental importância para impedir a progressão do processo evolutivo do carcinoma escamoso. Em nossa casuística, a citologia mostrou-se mais eficaz que a colposcopia na detecção destas lesões. A melhor correlação cito-histológica em nossa amostra foi verificada exatamente nas lesões da alto grau (79\%). Os critérios citológicos e histológicos nesta categoria são bem estabelecidos e de fácil reprodutibilidade ${ }^{23}$. Todavia, nem sempre uma boa correlação citohistológica nesta categoria diagnóstica tem sido relatada ${ }^{24,26}$.

Em resumo, pode-se concluir, a partir da análise dos resultados do presente estudo, que a associação do método colposcópico ao citológico é eficaz na detecção das alterações neoplásicas do colo do útero, sendo a correlaçãa cito-colpo-histológica de fundamental importância na identificação das categorias de maior dificuldade diagnóstica, como nas lesões de baixo grau. Este último fator é de importância no direcionamento do esforço de uniformização de critérios entre os clínicos e patologistas, lembrando que a colposcopia deve sempre ser indicada quando houver suspeita clínica de câncer, mesmo quando a citologia for negativa.

\section{Summary}

Sensibility and specificity of cytology and colposcopy exams with the histological evaluation of cervical intraepithelial lesions.

OBJECTIVE. To evaluate the correlation of cytopathological and colposcopycal diagnosis with the histopathological analysis frombiopsy.

METHODS. 80 patients from the colposcopy ambulatory of the Hospital e Maternidade Santa Brígida de Curitiba, PR, were selected. Those patients were sent to colposcopy under the following criteria: I) previous abnormal cytopathological exams, 2) Indicative clinical data or, 3) Suspected lesions on gynecologicalexam. The statistical significance analysis of the results was done using the chi-square test. Sensibility, specificity, positive predictive value (PPV) and negative predictive value (NPV) were also determined.

RESULTS. The patients'meanagewas 30.2 $( \pm 10.9)$. Cytopathological capability of identifyinglesions was 50\% when compared to histology. Its specificity was $77 \%$, the sensibility $41 \%$, the PPV $74 \%$, and the NPV $45 \%$. Colposcopy capability of identifying lesions was $50 \%$. Its sensibility, specificity, PPV and NPV were 96\%, 19\%, 65\% and $75 \%$ respectively. The two methods associated were capable of identifying 63\% of the lesions.

Conclusions. Cytopathology was a high specificity exam, while colposcopy from those selected patients had a high sensibility. Colposcopy association with cytopathological screening, on those selected patients, significantly raises the diagnostic accuracy of cervical cancer precursor lesions. [Rev Assoc Med Bras 2002; 48(2): 140-4]

Keywords: Cervical intraepithelial neoplasia. Cytopathology. Pap smear. Colposcopy. Comparative study. Histology. 


\section{REFERÊNCIAS}

I. Wallin KL, Wiklund F, Angstrom T, Bergman F, Stendahl $U$, Wadell $G$, et al. Type-specific persistence of human papillomavirus dna before the development of invasive cervical cancer. N Engl J Med 1999; 34 | : 633-8.

2. Manos MM, Kinney WK, Hurley LB. Identifying women with cervical neoplasia. JAMA 1999; 281:1605-10.

3. National Cancer Institute. The 1988 Bethesda system for reporting cervical/vaginal cytological diagnoses. JAMA 1989; 262:93।-4.

4. Van Niekerk WA, Dunton CJ, Richart RM, Hilgarth M, Kato $\mathrm{H}$, Kaufman $\mathrm{RH}$, et al. Colposcopy, cervicography, speculoscopy and endoscopy. International Academy of Cytology Task Force summary. Diagnostic cytology towards the 2Ist century: an international expert conference and tutorial. Acta Cytol 1998; 42:33-49.

5. Spuhler S, Sauthier P. Current indications for colposcopic examination. J Gynecol Obstet Biol Reprod (Paris) 1993; 22:722-8.

6. Allahverdian V, Valaitis J, Kalis $\mathrm{O}$, Pearlman $\mathrm{S}$. Cytology and colposcopy in the diagnosis and management of outpatients with cervical intraepithelial neoplasia. J Reprod Med I 980;24: I -4.

7. Kurman RJ, Henson DE, Herbst AL, Noller KL, Schiffman $\mathrm{MH}$. Interim Guidelines for management of abnormal cervical cytology. JAMA 1994; 27|: | 866-9.

8. McCord ML, Stovall TG, Summitt RL Jr, Ling FW. Discrepancy of cervical cytology and colposcopic biopsy: is cervical conization necessary? Obstet Gynecol 199/; 77:7 I 5-9.

9. Horn LC, Kuhndel K, Bilek K. Histopathologic correlates of false-positive cytologic findings in the uterine cervix. Geburtshilfe Frauenheilkd 1995; 55:425-30.
10. Heatley MK, Bury JP. The correlation between the grade of dyskaryosis on cervical smear, grade of cervical intraepithelial neoplasia (CIN) on punch biopsy and the final histological diagnosis on cone biopsies of the cervix. Cytopathology 1998; 9:93-9.

I I. Stafl A, Wilbanks G. International terminology of colposcopy. Cervix 1991; 9:91.

12. Mossetti C, De Palo G. A colposcopia ontem e hoje In: De Palo G, editor. Colposcopia e patologia do trato genital feminino inferior. 2a. ed. Rio de Janeiro: Editora Médica e Científica; 1991. p.37-63.

13. Ibrahim SN, Krigman HR, Coogan AC, Wax TD, Dodd LG, Bentley RC, et al. Prospective correlation of cervicovaginal cytologic and histologic specimens. Am J Clin Pathol 1996; 106:319-24.

14. Parham DM, Wiredu EK, Hussein KA. The cytological prediction of cervical intraepithelial neoplasia in colposcopically directed biopsies. Cytopathology 1991; 2:285-90.

15. Joseph MG, Cragg F, Wright VC, Kontozoglou TE, Downing P, Marks FR. Cyto-histological correlates in a colposcopic clinic: a I-year prospective study. Diagn Cytopathol 1991; 7:477-81

16. Korkolopoulou P, Kolokythas C, Kittas C, Alevritou H, Pavlakis K. Correlation of colposcopy and histology in cervical biopsies positive for CIN and/or HPV infection. Eur J Gynaecol Oncol 1992; 13:502-6.

17. Jesien U, Madej JG. The role of colposcopy in evaluation of the cervix for unexpectedly positive pap smears. Przegl Lek 1999; 56:17-9.

18. Maziah AM, Sharifah NA, Yahya A. Comparative study of cytologic and colposcopic findings in preclinical cervical cancer. Malays J Pathol |99|; |3: 105-8.
19. Chow VT, Tay SK, Tham KM, Lim Tan SK, Bernard HU. Subclinical human papillomavirus infection of the male lower genital tract: colposcopy, histology and DNA analysis. Int J STD AIDS | 99|; 2:41-5.

20. Bigrigg MA, Codling BW, Pearson P, Read MD, Swingler GR. Colposcopic diagnosis and treatment of cervical dysplasia at a single clinic visit. Experience of low-voltage diathermy loop in 1000 patients. Lancet 1990; 336:229-31.

21. Pearlstone AC, Grigsby PW, Mutch DG. High rates of atypical cervical cytology: occurrence and clinical significance. Obstet Gynecol 1992; 80:191-5.

22. Skehan M, Soutter WP, Lim K, Krausz T, Pryse DJ. Reliability of colposcopy and directed punch biopsy. Br J Obstet Gynaecol 1990; 97:811-6.

23. Brown FM. LSIL biopsies after HSIL smears. Correlation with high-risk HPV and greater risk of HSIL on follow-up. Am J Clin Pathol 1999; 1 12:765-8.

24. Lonky NM. The clinical significance of the poor correlation of cervical dysplasia and cervical malignancy with referral cytologic results. Am J Obstet Gynecol 1999; 181:560-6.

25. Papanicolaou GN, Traut HF. The diagnostic value of vaginal smears in the carcinoma of the uterus. Am J Obstet Gynecol I 94 I; 42: 1 93-206.

26. Collaço LM, Tuon FF, Soares MF, Totsugui J, Torres LF, Pinto AP. Correlação cito-histológica em 326 pacientes submetidas a cirurgia de alta freqüência (CAF) no programa de Prevenção de Câncer Ginecológico do Estado do Paraná. J Bras Patol 2000; 36:191-7.

Artigo recebido: 10/01/2001

Aceito para publicação: 22/02/2002

\title{
À BERA DO LETO
}

\author{
Envie sua contrbuição para esta nova seção da RAMB, \\ que apresenta perguntas com respostas objetivæs \\ sobre condutas práticas. Sua colaboração é \\ muito importante para nós.
}

\section{Ramb - Rua São Carlos do Pinhal, 324 - CEP 01333-903 São Paulo - SP - Tel.: (11) 32666800 - ramb@amb.orgbr}

